\title{
Development of Image Processing Technique for Detection of the Rescue Target in the Marine Casualty
}

\author{
T.Sumimoto*, K.Kuramoto*, S.Okada**, H.Miyauchi**, M.Imade ${ }^{* *}$, H.Yamamoto*** \\ and T.Kunishi**** \\ *Maritime Safety Academy. 5- 1 Wakaba-cho, Kure, Hiroshima 737, JAPAN \\ **Chugoku National Industrial Research Institute. 2-2-2 Hiro-suehiro, Kure, \\ Hiroshima 737-01, JAPAN \\ *** Okayama University. 3-1-1 Tsushima-naka, Okayama 700, JAPAN \\ ****Interface Co. Ltd. 1-1-25 Kaniya, Hiroshima 732, JAPAN
}

\begin{abstract}
When a marine casualty occurs, the detection of the rescue target such as life rafts depends on the visual search of human's eye. It is predicted, however, that human eye sometimes loses its sight and ability of detection falls down owing to the long flight and the nasty weather. For a practical purpose of the prompt rescue of human life, development of searching support system in place of human eye is surely required. To realize the detection of the small rescue target in the wide sea, we propose a new searching system using image processing techniques based on the color information.

The searching system consists of a visual sensor, an equipment of image processing and a knowledge database. Integration of these parts enables us to detect the rescue target accurately no matter how bad the condition is. At the first step of our study, we attempt the development of image processing techniques to detect accurately the rescue target under the various weather conditions.
\end{abstract}

\section{Introduction}

The automation of weather or typhoon forecast, topographical investigations of sea bottom and so on has been advanced by the developments of image processing, pattern recognition and knowledge engineering technologies. However, the detection of the rescue target in a marine casualty such as shipwreck depends on the visual search by man as yet. Human eyes sometimes lose its sight because of the long flight and the wide views under the various weather conditions. To detect the small target in the wide sea, a binocular telescope is usually employed for the magnification.

In that case, the range of vision for searching becomes narrow and the possibility of oversight will increase. Moreover, the ratio $\mathrm{S} / \mathrm{N}$ between the target and the background (sea surface) decreases due to the sunshine reflections, cloud shadows and white crested waves. These factors trouble us to detect the small targets. In order to carry out the prompt rescue of human life, development of searching support system using image processing techniques in place of the human eye is surely required. On the other hand, marine sports such as a yacht race or a cruising are popularized and simultaneously marine accidents arise along with it. For example, we remember clearly that in 1991 twice shipwreck of "Taka" and "Marine marine" were arisen at the Pacific Ocean. The problems of image processing techniques for detection of the rescue target are summarized in the following. One is the detection 
accuracy, that is, a very small target corresponds to only one or two pixel in the picture must be detected in the wide views over the sea. The other is the processing speed, that is, huge image data must be analyzed in a real time manner. One of the effective ways to acquiring the image of specific target accurately in wide views is to employ a composite image sensor system we have proposed before [1] ,

[2] , [3]. However, this system has been developed for manufacturing or construction in a production field and its range of vision was only $1 \mathrm{~m} \times 1 \mathrm{~m}$ area.

Moreover, it was developed assuming that the contrast against background is always high and the target is a size or a position of machine parts. Then, two problems mentioned above cannot be solved by means of the conventional image sensor system.

In this paper, we attempt to develop image processing techniques for detection of the rescue target under the various weather conditions. A curtailment of the image data and an improvement of the processing speed are expected. At the first step of our study, we attempted to extract the image data of the rescue target with the orange color in an experimental sea.

\section{System Configuration}

As is already mentioned, it is very hard to detect the small rescue target such as life crafts in the wide sea area where the ratio of $S / N$ between the rescue target and the background sea is extremely inferior. For the prompt rescue of human life, we propose a new searching system by paying attention to the color information. Figure 1 shows the system configuration for detection of the rescue target. The searching system consists of three processes as below.

(1) The first step is to attain the candidate of rescue target in the wide sea area : The image data obtained through the visual sensor such as the TV camera on the airplane is divided into the three primary colors (Red,Green and Blue). In the case of the searching by airplane, the rescue target becomes very small and background noise such as the sunshine

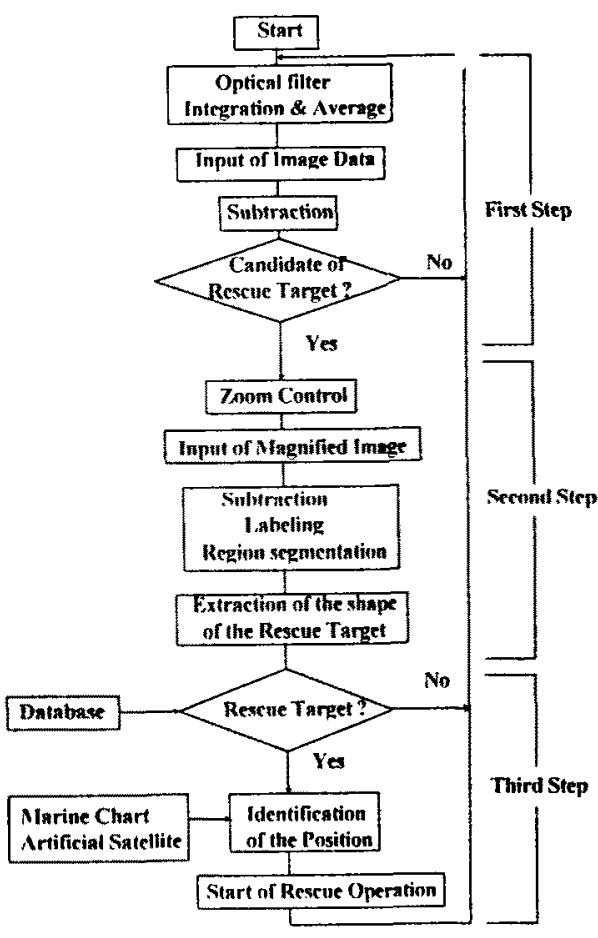

Fig. 1 System configuration 
reflections, cloud shadows and white crested waves usually exists. Here, we need to improve $\mathrm{S} / \mathrm{N}$ ratio in the image data.

(2) The second step is to acquire the magnified image by a zoom control and to extract the characteristics of rescue target : In order to avoid an overlook, the threshold value for judgment in the first step must be set up at low level. This means that a considerable error exists in this step. Then, a strict judgment whether the candidate involve the rescue target or not is necessary. The image in which the rescue target seems to exists is magnified by a zoom lens and a fresh image data is acquired. After the same subtraction between the $R$ and $G$ bands, we can extract the shape of the target and obtain the characteristics of the target from the binary image.

(3) The third step is to apply the knowledge database : In order to begin the correct rescue operation, it is necessary to determine the kind of the rescue target by collating the shape or color data obtained in the second step with the knowledge database and to determine the position of the rescue target by using the information of the marine chart or the artificial satellite. Here, characteristics of the rescue target (for example, kind, size or shape) must be registered at the knowledge database in advance. If the rescue target exists in the candidate, rescue operation begins immediately. If the rescue target dose not exist, the procedure is returned back to the initial state and the same process is carried out again.

We already attempted to extract the image data of the rescue float with orange color and recognized the shape of the rescue float on the magnified image ( the second step ) [4], [5] . To realize such searching system for detection of a small rescue target under various weather conditions, the development of a new type image sensor system in real time is undoubtedly necessary. Therefor, we attempt the development of the image processing technique of image data acquired under various weather conditions to detect surely the candidate of the rescue target in the wide sea. The third step (construction of a knowledge database) are unsolved subjects in the future and are not referred in this work.

\section{Model Experiment}

In the case of the rescue in the Pacific Ocean, the rescue party watches the sea surface at $5.4 \mathrm{~km}$ beyond from an airplane flying at $300 \mathrm{~m}$ height with a speed of 100 $\mathrm{m} / \mathrm{s}$ as shown in Fig.2. It is called that the white yacht with $10 \mathrm{~m}$ is the limit of visual search by human eye because of the optical absorption by gas, scattering by air, low contrast between target and background ( $\mathrm{S} / \mathrm{N}$ ratio), decrease of moving eyesight in high altitude and so on [6], [7] . So, small targets such as life rafts or floats etc. cannot be recognized. In the actual image acquired in the rescue activities the rescue target is very small and the images acquired under various weather conditions have background images such as ships, cloud and white crested waves. Therefor, in the first step we need to extract only the images of the rescue target.

As an example, let us suppose the rescue in the Pacific Ocean as is already 


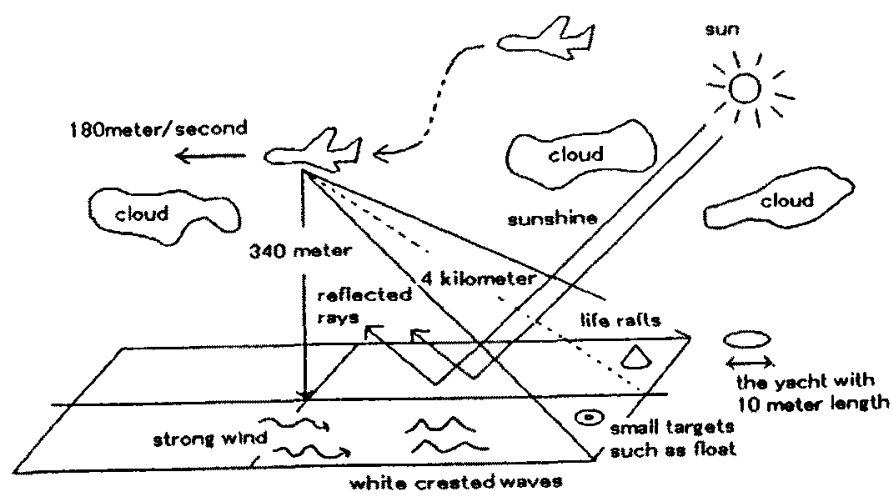

Fig. 2 mustration of searching activities

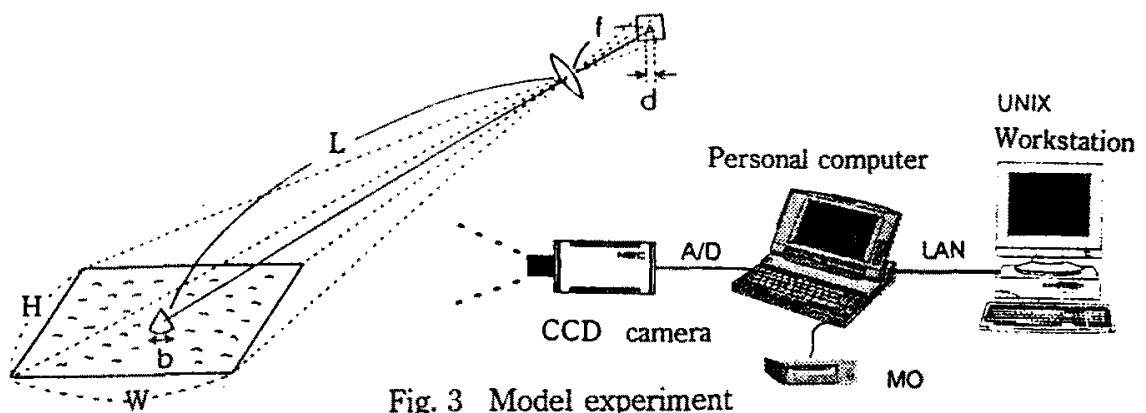

mentioned in section 1 . When we take a picture of yacht with $10 \mathrm{~m}$ at $5.4 \mathrm{~km}$ ahead through the TV camera( $1 / 2$ inch CCD image sensor) with 525 scanning lines and the $35 \mathrm{~mm}$ focal length as shown in Fig. 3, a searching view becomes to be $987 \mathrm{~m}$ in horizontal and $741 \mathrm{~m}$ in longitude from the equations. And then the image data of the yacht have several scanning lines.

$$
\mathrm{W}=6.4 \mathrm{~L} / \mathrm{f}, \quad \mathrm{H}=4.8 \mathrm{~L} / \mathrm{f}
$$

where $\mathrm{L}(\mathrm{m})$ is the distance between the rescue target and TV camera, $W(m)$ the width of the acquired image, $\mathrm{H}(\mathrm{m})$ the highness of the image and $f(\mathrm{~mm})$ the focal length of lens.

In the present work, we make a simulation experiment for the recognition of the marine rescue target. As an example of the rescue target, we selected the buoy with orange color(the bottom diameter is $3 \mathrm{~m}$ and the highness is $3.5 \mathrm{~m}$ ) in the Yoshiura bay. Figure 4 shows the marine chart and Figure 5 shows the photograph of

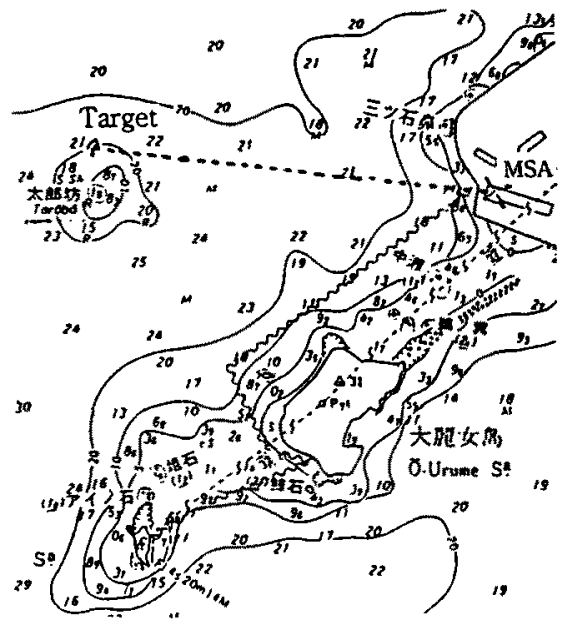

Fig. 4 Marine chart of Yoshiura bay 


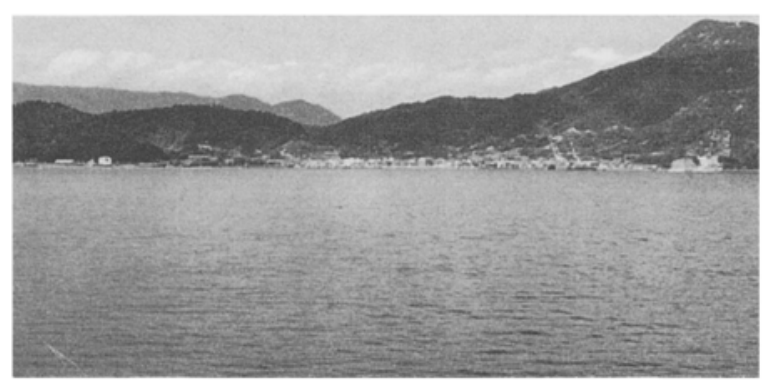

Fig. 5 Example of the rescue target

this buoy. The distance between the buoy and TV camera is about $650 \mathrm{~m}$ by calculation on the marine chart. In this scene the rescue target is scanned with $16 \mathrm{~cm}$ scanning resolutions.

Since the aim of the present study is to develop the image processing techniques in order to detect the rescue target under the various weather conditions, we attempt to acquire three kinds of image data of the rescue target under the different conditions. The first image data have the ship and the land as the background data. The second image data have the white crested waves. The third image data were taken at a rainy and fog weather and the range of vision is below $1000 \mathrm{~m}$. Then, we make an attempt to detect the rescue target from these image data recorded at the memories of the computer. To realize such searching system for detection of the rescue target under the various weather conditions, the development of a new type image sensor system in real time is undoubtedly necessary.

\section{Image Processing Techniques}

We propose a subtraction of the image data between the $R$ and $G$ band at the first stage of the searching system. As the color of rescue targets are orange, sunshine is absorbed in the green band and reflected in the red band. Namely, the image data of the rescue target have low gray levels in green band and high gray levels in red band. Therefore, only the image of target can be extracted by eliminating the image of the sea. At the same time, contrast of the target against the background ( $\mathrm{S} / \mathrm{N}$ ratio) can be improved and the probability of detection will progress. On the other hand, as the background noise such as reflections, clouds and waves is white, they have high gray level both in the red and green bands. It is notable that subtraction technique of the image data between the $R$ and $G$ bands is also effective method to exclude background white noise and other background data.

The image data of the rescue target is divided into the red band, the green band and the blue band and digitized into 256 levels with $512 \times 512$ size as shown in Fig. 3 . The image data are recorded on the magneto optical disk continuously. These image 
data are transmitted to the workstation for performing the processing of the image data with $C$ language.

\section{Result and Discussion}

(1) Case 1: The image data have the ship and the land as the background data.

The original image data which have been shown on the CRT of image processor is indicated in Fig.6(a), where the image data have the ship and the land. Figure 6(b) shows the subtracted image data between the $R$ and $G$ band. It is found that the ship and the land as the background image data is efficiently excluded and then the $S / N$ ration of the target against background is improved. As the noise reduction is often necessary to get a better result, median filter was employed to the present data.

(2) Case 2: The image data have the white crested waves.

The original image data have been shown in Fig.7(a). and Fig.7(b) shows the subtracted image data between the $R$ and $G$ band. The white crested waves is excluded efficiently.

(3) Case 3: The image data were taken at rainy and fog day.

In the original data the region of the rescue target becomes dark. It is considered

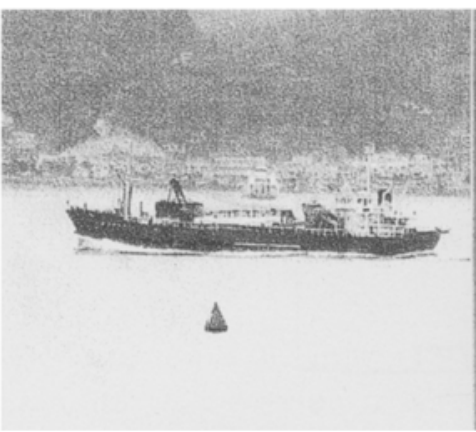

a) Original image data

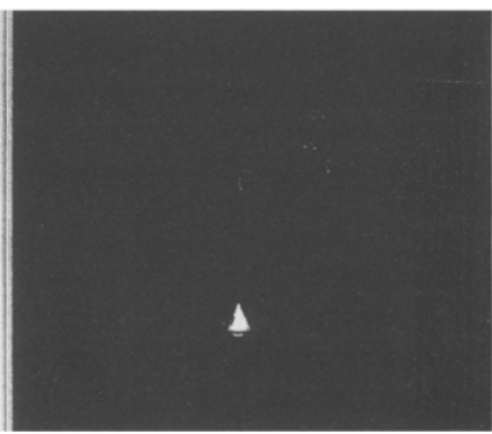

b) Result of image processing

Fig. 6 The image data having the ship and the land as the background data.

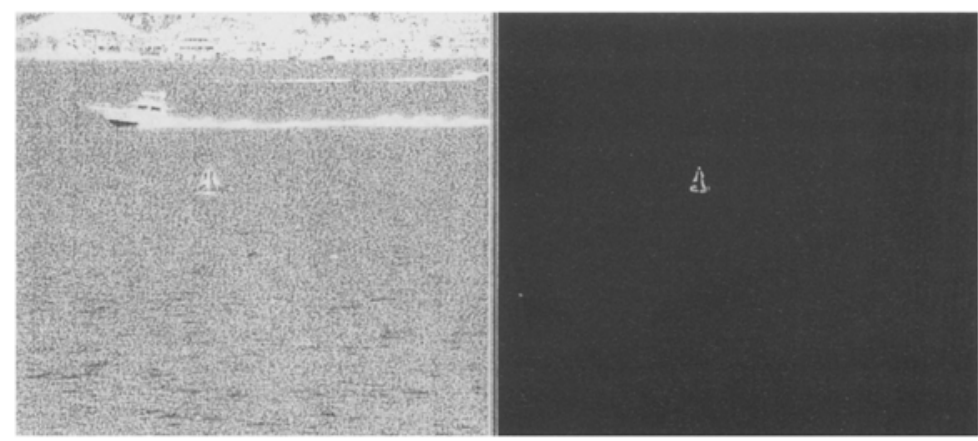

a) Original image data

b) Result of image processing

Fig. 7 The image data having the white crested waves. 
that the reflections of lights from the surface of the rescue target are absorbed and the reflections of lights with green spectrum from the sea surface are scattered by waters and vapours on the way. In the red band the grey levels of the region of the rescue target becomes low than that of the sea owing to absorption. In the green band the grey level of the region of the sea becomes high owing to scattering of green spectrum. Then, we cannot extract the rescue target by subtraction between the $R$ and $G$ band. In order to adjust the background levels in the two bands, we add a fixed grey level(20counts) to the red band and subtract between the $R$ and $G$ band as shown in Fig. 8. As the result of this method we can extract the rescue target as shown in Fig. 9.

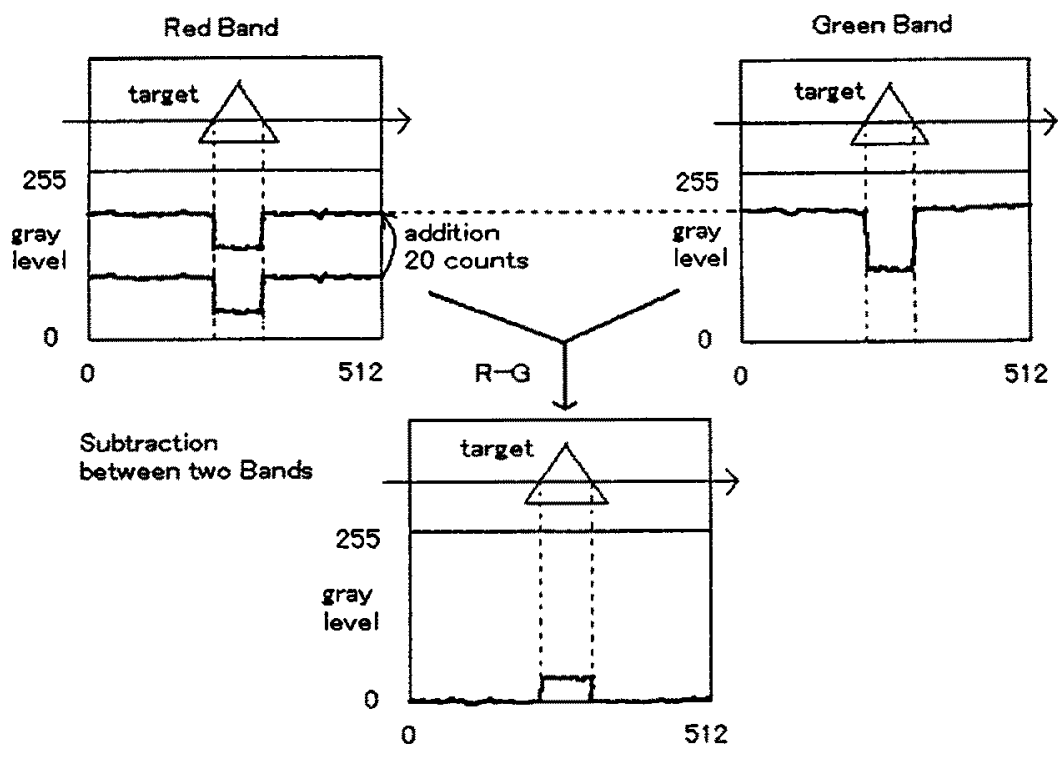

Fig. 8 Illustration of subtraction between two bands of images acquired under the rainy and fog weather

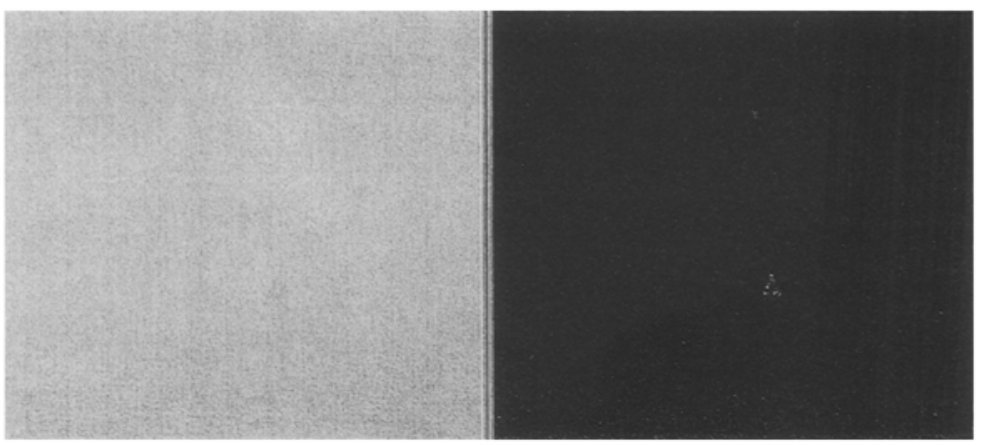

a) Original image data

b) Result of image processing

Fig. 9 The image data were taken at rainy and fog day. 


\section{Conclusion}

For the purpose of the prompt rescue of a human life, the searching support system using image processing technique was proposed. In concretely, we have proposed a new searching system including the techniques of extraction based on color information to detect the rescue target from the image data acquired under the various weather conditions. At the first step of the study, we deal with the rescue target in an experimental sea and significant results are obtained as follows.

(1) The background image data are efficiently excluded and the $S / N$ ration of the target against background is improved by the subtraction of the image data between the red band and green band.

(2) The detection of the rescue target from the image data acquired under the rainy and fog weather is performed effectively by means of the image processing techniques.

It is concluded, therefore, that the image processing technique proposed in this study is an effective method for detection of the rescue target under various weather conditions. To realize the searching system for the actual rescue activity, further studies are desired; the construction of the control system of the visual sensor with high resolution and high speed and also the knowledge database system of color and shape information in rescue targets.

\section{References}

[1] Okada S., Sumimoto T., Miyauchi H. and Yamamoto H. : Dimension and Shape Measurements with High Accuracy Using Composite Image Sensors: T.IEE Japan Vol.110-D,No.3,(1190)212-217.

[2] Okada S., Sumimoto T., Miyauchi H. Imade M. and Yamamoto H. : Shape Inspection of 3-D Objects Using Time-Coded Pattern Projection and Newly Developed Image Sensor Systems : Proceedings of IAPR, Japan(1990)55-58.

[ 3 ] Sumimoto T., Okada S., Miyauchi H., Imade M. and Yamamoto H.: Object shape measurement by a combination of area and line sensor, Proceedings of IMEKO 12th, China,(1991)889-894.

[4] Sumimoto T., Kuramoto K., Okada S., Miyauchi H., Imade M., Yamamoto H and Kunishi T.:Detection of the rescue target in the marine casualty using image processing techniques, The second APIS/IMAC, X'iam China(1993)341-345.

[ 5 ] Sumimoto T., Kuramoto K., Okada S., Miyauchi H., Imade M., Yamamoto H. and Kunishi T.: A new rescue system in the marine casualty using image processing techniques, Proceedings of 5th ISME Yokohama '95(1995)250-255.

[6] Rescue Manual \$2-3 Search and Rescue : Maritime Safety Agency.

[7] National Search and Rescue Manual : U.S.Cost Guard (1986). 